\title{
A Stable Floating Non-Foster Circuit
}

\author{
Farzad Tofigh*, Richard W. Ziolkowski* \\ *University of Technology Sydney, Australia \\ email:farzad.tofigh@uts.edu.au
}

\begin{abstract}
Non-Foster elements have attracted considerable interest because of their role in enabling wide-band electrically small antennas and metamaterials. However, the potential instability of these elements continues to be an going challenge to their realization and, hence, practical acceptance. In this paper, we present a stable floating negative impedance converter (NIC) element using a RLC (resistor-inductor-capacitor) based subcircuit as the load.
\end{abstract}

Index Terms-Foster's theory; Non-Foster circuits; negative impedance; negative impedance converter (NIC); stability

\section{INTRODUCTION}

Foster's reactance theorem states that the reactance of a passive network monotonically increases as the frequency increases. Consequently, circuits whose reactance decreases as the frequency increases are known as non-Foster circuits. Typically, they are represented as negative capacitors or negative inductors. The impact of having such a circuit is its ability to help overcome basic physics restrictions, such as Chu's limit [1], that are associated with passive devices. Non-Foster elements have led to the realization of broad bandwidth electrically small antennas [2]-[4], to the control of the dispersion of metamaterials [5], [6], and to the broadening of the bandwidth of cloaking devices [7].

Negative impedance converters (NICs) and negative impedance inverters (NIIs) are active circuits that transform passive reactances into non-Foster elements. Linvill's crosscoupled transistors [8], positive-feedback operational amplifiers, and negative resistor circuits are promising configurations that provide negative impedance at their output ports. The main difficulty with these configurations is their use of positive feedback for the purpose of manipulating their voltage-current relations, which can lead to the instability of their circuits [9]. Consequently, the main challenge in the design and realization of any bandpass non-Foster circuit is ensuring that it is stable in the frequency interval of interest [10].

Stearns [10] suggests that a bandpass non-Foster circuit exhibiting unconditional stability can be achieved using a combination of ideal (like sign) $\pm R, \pm L$ and $\pm C$, elements as the load. This paper provides a realization of a stable floating NIC that is based on Linvill's design and that operates at $300 \mathrm{MHz}$ with the intent to replace an inductor in the nearfield resonant parasitic (NFRP) element of a metamaterialinspired electrically small antenna (ESA). It also investigates with a time domain analysis what effect the resistor has on the stability of that circuit.

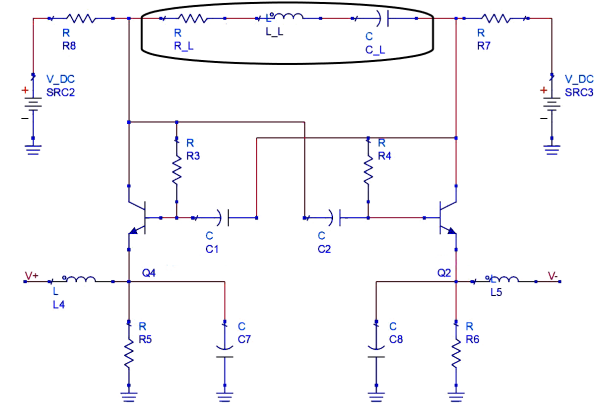

Fig. 1. Linvill-based NIC with cross-coupled transistors giving positivefeedback.

TABLE I

PARAMETER VALUES OF THE PROPOSED NIC CIRCUIT.

\begin{tabular}{|c|c|c|c|c|c|}
\hline Parameter & Value & Parameter & Value & Parameter & Value \\
\hline$L_{4}$ & $33 n H$ & $R_{5}$ & $75 \Omega$ & $C_{7}$ & $5.4 p F$ \\
\hline$L_{5}$ & $33 n H$ & $R_{6}$ & $75 \Omega$ & $C_{8}$ & $5.4 p F$ \\
\hline$C_{1}$ & $22 p F$ & $C_{2}$ & $22 p F$ & $R_{3}$ & $300 \Omega$ \\
\hline$R_{4}$ & $300 \Omega$ & $R_{7}$ & $300 \Omega$ & $R_{8}$ & $300 \Omega$ \\
\hline
\end{tabular}

\section{NON-FOSTER CIRCUIT WITH A RLC LOAD}

The proposed Linvill-based NIC circuit is based on a pair of cross-coupled BJT transistors, AT41533, that are connected to a positive load. As noted, this load consists of a capacitor $\left(C_{L}\right)$, an inductor $\left(L_{L}\right)$, and a resistor $\left(R_{L}\right)$. The ADS model of the proposed circuit is illustrated in Fig. 1. The bias network, which consists of a $9 \mathrm{~V}$ dc source and lumped element $\mathrm{L}, \mathrm{C}$, and $\mathrm{R}$ values, was optimized numerically. The final critical parameter values are listed in Table I.

The desired structure is designed to replace a specified set of inductor values at particular frequencies derived from HFSS simulations of the NFRP-based ESA. Thus, the designed circuit must provide the desired equivalent reactance and near zero resistance (to maintain high radiation efficiency). Setting $C_{L}=2.2 p F, L_{L}=120 n H$, and $R_{L}=100 \Omega$ leads us to the desired reactance and resistance values. Fig. 2 shows the reactance and resistance of the designed element associated with its ports $V+$ and $V-$.

\section{STABILITY ANALYSIS OF THE NON-FOSTER ELEMENT}

Methods of stability analysis in active circuit design such as Rollet's proviso are usually not valid for non-Foster circuits [10]. On the other hand, transient analyses are robust tech- 


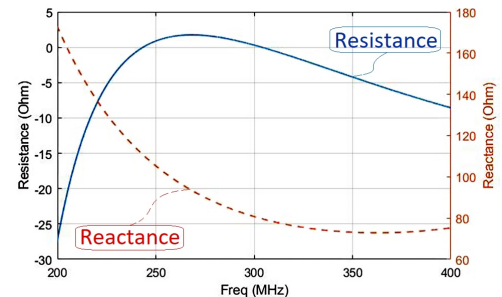

Fig. 2. Resistance and reactance of the circuit with $R_{L}=100 \Omega$.

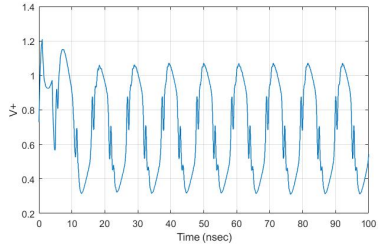

(a1)

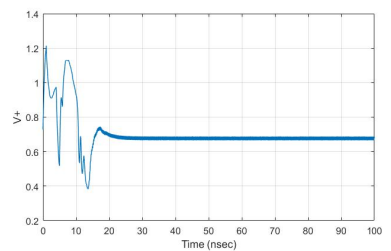

(b1)

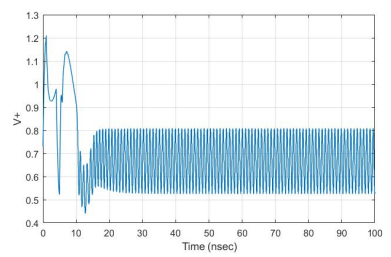

(c1)

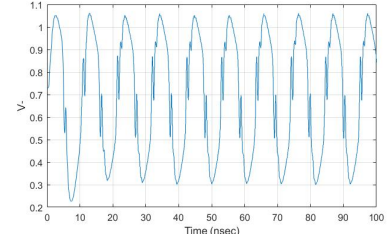

(a2)

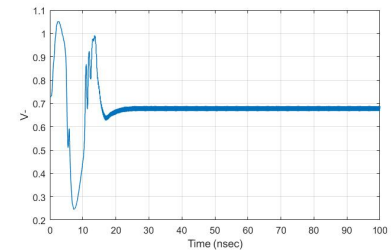

(b2)

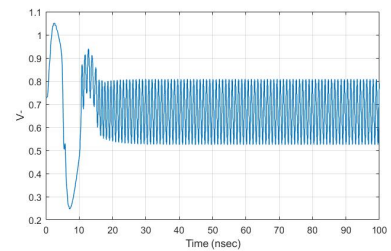

(c2)
Fig. 3. (a*) Transient analysis on ports $V+$ and $V$ - with no load resistor. (b*) Transient analysis on ports $V+$ and $V$ - with $R_{L}=100 \Omega$. (c*) Transient analysis on ports $V+$ and $V$ - with $R_{L}=200 \Omega$.

niques to analyze the voltage and current responses to a pulse excitation [11], [12].

As mentioned, the inclusion of a load resistor to a LC load combination can offer more stable non-Foster circuits. Fig. 3 shows the voltage response on ports $V+$ and $V-$ in the time domain for the proposed non-Foster element for various values of the load resistor. As can be seen, the addition of the load resistor can increase the stability of the circuit. However, there is a limit to the size of the resistance. The circuit is stable when $30 \Omega<R_{L}<200 \Omega$.

Furthermore, the resistor can affect the circuit input resistance as seen from ports $V+$ and $V-$. Fig. 4 represents the input resistance for various values of the load resistor. The NIC's resistance decreases as the load resistor increases. For instance, the resistance is $0.11 \Omega$ at $300 \mathrm{MHz}$ when $R_{L}=100 \Omega$. With

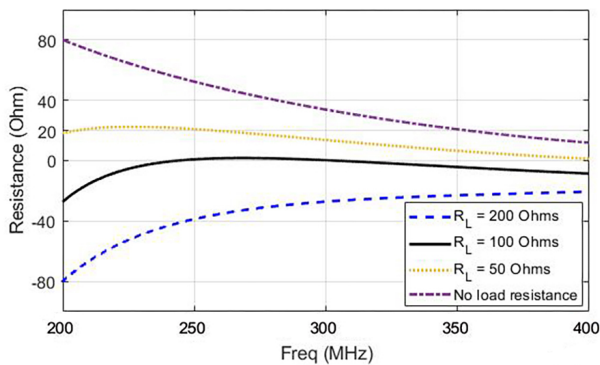

Fig. 4. Resistance of the circuit with $R_{L}=200 \Omega, R_{L}=100 \Omega, R_{L}=50 \Omega$ and without load resistance.

$R_{L}=0 \Omega$ and $R_{L}=200 \Omega$, the resistance drops, respectively, from $37 \Omega$ to $-27 \Omega$, which causes the unstable results in Figs. 3 (c1) and (c2).

\section{CONCLUSION}

In this paper we investigated the effect of a load resistor, as an added element to a series LC load combination, on the stability of the resulting NIC circuit. It was shown that adding a resistor to the load can increase the stability of the circuit while manipulating the resistance of the circuit. It was also shown that the circuit is only stable within a specific range of the resistor value. Introducing a resistance outside of that range leads to an unstable element.

\section{REFERENCES}

[1] L. J. Chu, "Physical limitations of omni-directional antennas," Journal of applied physics, vol. 19, no. 12, pp. 1163-1175, 1948.

[2] A. M. Elfrgani and R. G. Rojas, "Successful realization of non-foster circuits for wide-band antenna applications," in 2015 IEEE MTT-S International Microwave Symposium. IEEE, 2015, pp. 1-4.

[3] N. Zhu and R. W. Ziolkowski, "Broad-bandwidth, electrically small antenna augmented with an internal non-foster element," IEEE Antennas and Wireless Propagation Letters, vol. 11, pp. 1116-1120, 2012.

[4] C. R. White and C.-T. Tsen, "On the stability of non-foster monopole antenna arrays," in 2013 IEEE MTT-S International Microwave Symposium Digest (MTT). IEEE, 2013, pp. 1-4.

[5] J. Long, M. M. Jacob, and D. F. Sievenpiper, "Broadband fast-wave propagation in a non-foster circuit loaded waveguide," IEEE Transactions on Microwave Theory and Techniques, vol. 62, no. 4, pp. 789-798, 2014.

[6] K. Rajab, Y. Fan, and Y. Hao, "Characterization of active metamaterials based on negative impedance converters," Journal of Optics, vol. 14 no. 11, p. 114004, 2012.

[7] P.-Y. Chen, C. Argyropoulos, and A. Alù, "Broadening the cloaking bandwidth with non-foster metasurfaces," Physical review letters, vol. 111, no. 23, p. 233001, 2013.

[8] J. G. Linvill, "Transistor negative-impedance converters," Proc. IRE, vol. 41, no. 6, pp. 725-729, Jun. 1953.

[9] J. Brownlie, "On the stability properties of a negative impedance converter," IEEE Transactions on Circuit Theory, vol. 13, no. 1, pp. 98-99, 1966.

[10] S. D. Stearns, "Incorrect stability criteria for non-foster circuits," in Proceedings of the 2012 IEEE International Symposium on Antennas and Propagation, July 2012, pp. 1-2.

[11] _ "Non-foster circuits and stability theory," in 2011 IEEE International Symposium on Antennas and Propagation (APSURSI), July 2011 pp. 1942-1945.

[12] A. Elfrgani, R. Moussounda, and R. Rojas, "Time-domain stability analysis/design of negative impedance inverters and converters," in 2013 IEEE MTT-S International Microwave Symposium Digest (MTT). IEEE, 2013, pp. 1-4. 